\title{
Abundance and host associations of parasitoids attacking frugivorous drosophilids on Iriomote-jima, a subtropical island of Japan
}

\author{
Biljana NOVKOViĆ, Ayako OIKAWA, Yusuke MURATA, Hideyuki MitSUI and Masahito T. KiMURA \\ Graduate School of Environmental Earth Science, Hokkaido University, Sapporo, Hokkaido 060-0810, Japan; \\ e-mail: biljanan@ees.hokudai.ac.jp
}

Key words. Hymenoptera, Braconidae, Asobara, Figitidae, Leptopilina, Diptera, Drosophilidae, Drosophila, parasitoid, host association, resistance, virulence

\begin{abstract}
To understand the factors governing the diversity, abundance and host associations of parasitoids attacking frugivorous drosophilid flies on Iriomote-jima, a subtropical island of Japan, we monitored parasitism on several occasions over the period 2003-2009. Fifteen drosophilid and 12 parasitoid species were recorded. Three species of Drosophila, D. bipectinata, D. albomicans and D. takahashii, bred abundantly in banana baits, though their abundance varied between years and seasons. Frequent parasitoid species were Asobara japonica, A. pleuralis (Braconidae), Leptopilina ryukyuensis and L. pacifica (Figitidae). L. victoriae was recorded only in December 2003. In addition, host acceptance and host suitability of the four most frequently recorded parasitoid species were studied in the laboratory. Most parasitoid and drosophilid species showed species-specific associations with more than one antagonist species, suggesting that they have been subjected to complex coevolutionary interactions. In addition, host range of most of the parasitoid species included one of the three major Drosophila species, suggesting that the abundance of potential hosts is one of the factors determining the evolution of parasitoid host use.
\end{abstract}

\section{INTRODUCTION}

Field studies that focus on host-parasitoid relations and the changes and variations that occur in host-parasitoid communities in space and time, contribute a great deal to our understanding of host-parasitoid coevolutionary interactions. These are particularly important for analyzing population dynamics of both hosts and their parasitoids, and essential when assessing the evolution of host resistance and parasitoid virulence in multi-species hostparasitoid systems.

Frugivorous drosophilids are attacked mainly by larval and pupal parasitoids and successful parasitism results in the death of the host. Pupal parasitoids are either ecto- or endoparasitoids and tend to be generalists, attacking a wide range of hosts. Larval parasitoids, on the other hand, are endoparasitoids that interact with the hosts' immune system and tend to have a narrow host range (Carton et al., 1986; Fleury et al., 2009).

The effect parasitoids have on host mortality is likely to exert a strong selective pressure on host resistance and parasitoid virulence. In order to escape parasitism, drosophilid hosts can mount immunological attacks against larval parasitoids. Parasitoids in return evolve ways to cope with host defenses, such as sticky eggs, virus-like particles and venom virulence factors (Fleury et al., 2009). Both host resistance and parasitoid virulence are thought to be graded traits, which form clines and mosaics in natural communities, based on local selective forces (Boulétreau \& Fouillet, 1982; Carton \& Nappi, 1991; Kraaijeveld \& van Alphen, 1994, 1995; Kraaijeveld et al., 1998; Kraaijeveld \& Godfray, 1999).
The majority of comprehensive field studies on the assemblage of drosophilid flies and their parasitoids were done in Europe (Jansen et al., 1988; Hardy \& Godfray, 1990; Allemand et al., 1999; Fleury et al., 2004; Wertheim et al., 2006). Most of these field studies and those carried out in North America (Hertlein, 1986) and Northern Africa (Carton et al., 1991), predominantly focused on two cosmopolitan drosophilids, Drosophila melanogaster Meigen and D. simulans Sturtevant, and their parasitoids Leptopilina heterotoma (Thompson), L. boulardi (Barbotin, Carton \& Kelner-Pillault) and Asobara tabida Nees von Esenbeck. These studies give insights into the structure and seasonal dynamics of host parasitoid communities, and address coevolution and competition in the respective regions.

Recent field surveys conducted in Japan are the first on native Asian drosophilid flies and their parasitoids (Yorozuya, 2006; Mitsui et al., 2007; Mitsui \& Kimura, 2010). Yorozuya (2006) reports the effects of parasitoids on mycophagous drosophilids in northern Japan in different seasons. Mitsui et al. (2007) present an overview of the distribution of hosts and their parasitoids. Mitsui \& Kimura (2010) concentrate on host parasitoid communities in central Japan, which are dominated by two parasitoid species, Asobara japonica Belokobylskij and Ganaspis xanthopoda (Ashmead).

There are several studies on the seasonal aspects of the drosophilid fauna on Iriomote-jima, a subtropical island of Japan (Okada, 1965; Takada \& Makino, 1981; Hirai et al., 2000), and some of the parasitoid species attacking Drosophila species occurring on this island are reported by Mitsui et al. (2007) and Novković et al. (2011). How- 


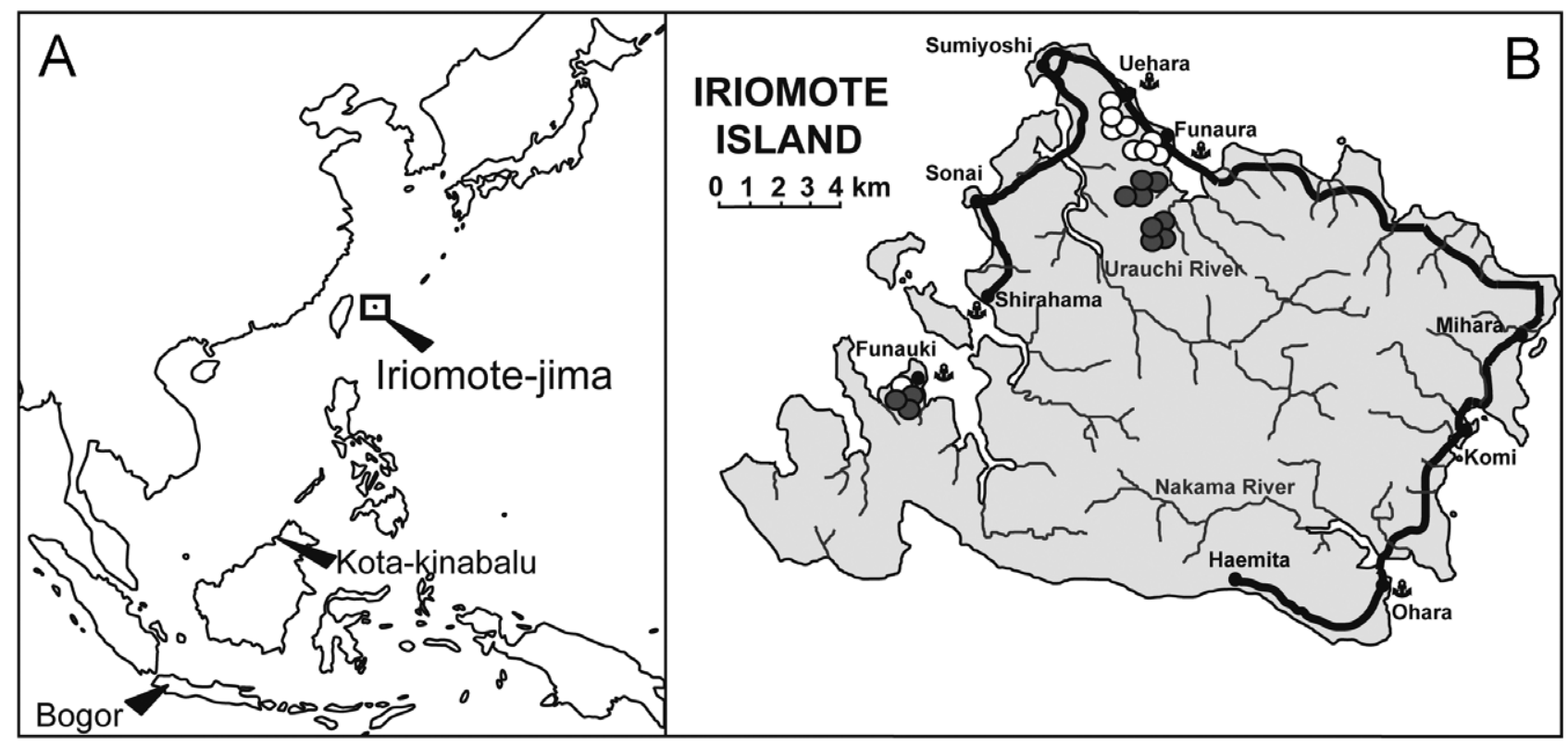

Fig. 1. A - location of Iriomote-jima and two other sites from which strains of D. bipectinata and L. victoriae were collected (Bogor and Kota-Kinabalu); B - map of Iriomote-jima with approximate locations of the traps. Traps in forested areas are indicated by grey and those in domestic areas by white circles.

ever, issues such as parasitoid seasonality, habitat preference, host acceptance and host suitability were not previously addressed.

Here, we report the results of a field survey conducted between 2003 and 2009 with the objective of determining the diversity, abundance and association of frugivorous drosophilids and their parasitoids on Iriomote-jima and of better understanding the factors that govern hostparasitoid associations. Additionally, we used laboratory experiments to study the host acceptance and host suitability for abundant drosophilid and parasitoid species. Based on these results we discuss the differences in the host-parasitoid communities recorded on Iriomote-jima, in central Japan and other previously surveyed regions.

\section{MATERIAL AND METHODS}

\section{Study site}

Our study area, Iriomote-jima $\left(24.2^{\circ} \mathrm{N}, 123.8^{\circ} \mathrm{E} ; 284.4 \mathrm{~km}^{2}\right)$, is a subtropical island located in the southern part of the Ryukyu archipelago (Fig. 1). Ninety percent of the island is covered by broadleaf evergreen forest and mangrove swamps, 34.3\% of which is in the Iriomote National Park. The maximum and minimum monthly mean temperatures on this island are approximately 28 (July) and $18^{\circ} \mathrm{C}$ (January), respectively. Temperatures occasionally reach as low as $7^{\circ} \mathrm{C}$ in winter.

\section{Field survey}

Parasitism was monitored on nine occasions over the period 2003 to 2009 using banana-baited traps. Traps containing 30-50 $\mathrm{g}$ of banana were set in forest and domestic environments. Domestic traps were set in Uehara and Funaura and the forest traps in the vicinity of a river in the forest south of Funaura. On

TABLE 1. Dates sampled, number of traps and bait used.

\begin{tabular}{|c|c|c|c|c|}
\hline \multirow{2}{*}{ Month sampled } & \multirow{2}{*}{ Date } & \multicolumn{2}{|c|}{ Number of traps } & \multirow{2}{*}{ Bait } \\
\hline & & Forest & Domestic & \\
\hline $2003 \mathrm{Jul}$ & 26-31 July & 5 & 5 & Banana \\
\hline 2003 Dec & 20-28 December & 10 & 6 & Banana \\
\hline 2007 Nov & 17-24 November & 8 & 8 & Banana \\
\hline 2007 Dec & 22-29 December & $8(+4)$ & $8(+1)$ & Banana \\
\hline $2008 \mathrm{Mar}$ & 26 March - 2 April & 8 & 8 & Banana \\
\hline 2008 Jun & 25-30 June & 8 & 8 & Banana \\
\hline 2008 Sep & 25-20 September & 8 & 8 & Banana \\
\hline 2009 May & 23-30 May & $\begin{array}{l}2 \\
2 \\
2\end{array}$ & $\begin{array}{l}2 \\
2 \\
2\end{array}$ & $\begin{array}{c}\text { Banana } \\
\text { Cherry } \\
\text { Mixed vegetables } \\
\text { (Figs) }\end{array}$ \\
\hline 2009 Sep & 21-27 September & $\begin{array}{l}2 \\
2 \\
2\end{array}$ & $\begin{array}{l}2 \\
2 \\
2\end{array}$ & $\begin{array}{c}\text { Banana } \\
\text { Cherry } \\
\text { Mixed vegetables }\end{array}$ \\
\hline
\end{tabular}


TABLE 2. Number of drosophilid pupae collected from banana-baited traps and their percentage parasitism by Asobara japonica (Aj), A. pleuralis (Ap), Leptopilina ryukyuensis (Lr), L. pacifica (Lp), L. victoriae (Lv), Ganaspis sp. IR1 (G1), Ganaspis xanthopoda $(\mathrm{Gx})$ and Tricopria sp. (Tr).

\begin{tabular}{|c|c|c|c|c|c|c|c|c|c|}
\hline & Pupae & $\mathrm{Aj}$ & Ap & $\mathrm{Lr}$ & Lp & $\mathrm{Lv}$ & G1 & Gx & $\mathrm{Tr}$ \\
\hline \multicolumn{10}{|l|}{2003 July } \\
\hline D. takahashii & 30 & 0 & 0 & 0 & 0 & 0 & 0 & 0 & 0 \\
\hline D. bipectinata & 392 & 0 & 0 & 0 & 0 & 0 & 0 & 0 & 0 \\
\hline D. albomicans & 235 & 0 & 0 & 0 & 0 & 0 & 1.3 & 0 & 0 \\
\hline D. daruma & 319 & 0 & 0 & 0 & 0 & 0 & 10.3 & 0 & 0 \\
\hline Undetermined & 3 & 0 & 0 & 0 & 0 & 0 & 0 & 0 & 0 \\
\hline \multicolumn{10}{|l|}{2003 December } \\
\hline D. takahashii & 938 & 7.5 & 0 & 0 & 0 & 0 & 0 & 0 & 0 \\
\hline D. bipectinata & 278 & 0 & 0.4 & 0 & 0 & 54.3 & 0 & 0 & 0 \\
\hline D. lacteicornis & 115 & 0 & & 2.6 & 0 & 0 & 0 & 0 & 0 \\
\hline D. ficusphila & 23 & 0 & 21.7 & 0 & 0 & 0 & 0 & 0 & 0 \\
\hline D. albomicans & 578 & 0 & 4.5 & 22.8 & 0 & 0 & 0 & 0 & 0 \\
\hline \multicolumn{10}{|l|}{2007 November } \\
\hline D. takahashii & 379 & 5.5 & 0 & 0 & 0 & 0 & 0 & 0 & 0 \\
\hline D. bipectinata & $640^{\mathrm{a}}$ & $3^{e}$ & 0 & 0 & 0 & 0 & 0 & 0 & 0 \\
\hline D. lacteicornis & 1 & 0 & 0 & 0 & 0 & 0 & 0 & 0 & 0 \\
\hline D. ficusphila & 1 & 0 & 0 & 0 & 0 & 0 & 0 & 0 & 0 \\
\hline D. albomicans & 218 & 0 & 14.7 & 0 & 0 & 0 & 0 & 0 & 0 \\
\hline D. ruberrima & 9 & 0 & 0 & 0 & 0 & 0 & 0 & 0 & 0 \\
\hline D. quadrilineata & 43 & 0 & 0 & 0 & 0 & 0 & 0 & 0 & 0 \\
\hline D. melanogaster & 29 & 0 & 0 & 0 & 0 & 0 & 0 & 0 & 0 \\
\hline \multicolumn{10}{|l|}{2007 December } \\
\hline D. takahashii & 330 & 13.6 & 0 & 0 & 0 & 0 & 0 & 0 & 0 \\
\hline D. bipectinata & $852^{\mathrm{b}}$ & 0.6 & 0 & 0 & 0 & 0 & 0 & 0 & 0 \\
\hline D. lacteicornis & 139 & 0.7 & 1.4 & 17.3 & 0 & 0 & 0 & 0 & 0 \\
\hline D. ficusphila & 37 & 2.7 & 0 & 8.1 & 0 & 0 & 0 & 0 & 0 \\
\hline D. albomicans & 1735 & 0 & 3.7 & 26.8 & 0 & 0 & 0 & 0 & 0 \\
\hline D. ruberrima & 20 & 0 & 0 & 0 & 0 & 0 & 0 & 0 & 0 \\
\hline D. quadrilineata & 152 & 0 & 0 & 13.2 & 0 & 0 & 0 & 0 & 0 \\
\hline D. melanogaster & 14 & 7.1 & 0 & 0 & 0 & 0 & 0 & 0 & 0 \\
\hline S. dorsocentralis & 99 & 0 & 0 & 0 & 0 & 0 & 0 & 0 & 0 \\
\hline Phortica magna & 5 & 0 & 0 & 0 & 0 & 0 & 0 & 0 & 0 \\
\hline \multicolumn{10}{|l|}{2008 March } \\
\hline D. takahashii & 487 & 16.2 & 0 & 0 & 0 & 0 & 0 & 0 & 0 \\
\hline D. lacteicornis & 2 & 0 & 0 & 0 & 0 & 0 & 0 & 0 & 0 \\
\hline D. ficusphila & 108 & 0 & 0 & 0 & 0 & 0 & 0 & 2.7 & 0 \\
\hline D. albomicans & 83 & 0 & 0 & 0 & 7.2 & 0 & 0 & 0 & 0 \\
\hline \multicolumn{10}{|l|}{2008 June } \\
\hline D. takahashii & 214 & 6.1 & 0 & 0 & 0 & 0 & 0 & 0 & 0 \\
\hline D. bipectinata & 94 & 0 & 0 & 0 & 0 & 0 & 0 & 0 & 0 \\
\hline D. ficusphila & 3 & 0 & 0 & 0 & 0 & 0 & 0 & 0 & 0 \\
\hline D. albomicans & 206 & 0 & 2.4 & 4.8 & 0 & 0 & 0 & 0 & 0 \\
\hline D. melanogaster & 20 & 0 & 0 & 0 & 0 & 0 & 0 & 0 & 0 \\
\hline S. bryani & 2 & 0 & 0 & 0 & 0 & 0 & 0 & 0 & 0 \\
\hline \multicolumn{10}{|l|}{2008 September } \\
\hline D. takahashii & 13 & 0 & 0 & 0 & 0 & 0 & 0 & 0 & 0 \\
\hline D. bipectinata & $3924^{\mathrm{c}}$ & 0 & 0 & 0 & 0 & 0 & 0 & 0 & 0 \\
\hline D. ficusphila & 3 & 0 & 0 & 0 & 0 & 0 & 0 & 0 & 0 \\
\hline D. albomicans & $1427^{\mathrm{d}}$ & 0 & 13.9 & 0 & 0 & 0 & 0 & 0 & 0.1 \\
\hline D. bocki & 2 & 0 & 0 & 0 & 0 & 0 & 0 & 0 & 0 \\
\hline \multicolumn{10}{|l|}{2009 September } \\
\hline D. takahashii & 23 & 0 & 0 & 0 & 0 & 0 & 0 & 0 & 0 \\
\hline D. bipectinata & 1819 & 0 & 0 & 0 & 0 & 0 & 0 & 0 & 0 \\
\hline D. albomicans & 273 & 0 & 1.8 & 33 & 0 & 0 & 0 & 0 & 0 \\
\hline D. quadrilineata & 1 & 0 & 0 & 0 & 0 & 0 & 0 & 0 & 0 \\
\hline
\end{tabular}

a included 37 individuals of $D$. ananassae; ${ }^{\mathrm{b}}$ included individuals of $D$. ananassae; ${ }^{\mathrm{c}}$ included 20 individuals of $D$. melanogaster;

${ }^{\mathrm{d}}$ included 14 individuals of $D$. ruberrima; ${ }^{\mathrm{e}}$ probably emerged from $D$. ananassae. 
TABLE 3. Number of parasitoids that emerged from drosophilid pupae collected from banana, cherry and mixed vegetable-baited traps and naturally occurring figs in May and September 2009. Asobara japonica (Aj), A. pleuralis (Ap), Leptopilina ryukyuensis (Lr), L. pacifica (Lp), Ganaspis sp. IR2 (G2), G. xanthopoda (Gx), Leptolamina sp. (Le), Spalangia sp. (Sp) and Tachinaephagus sp. (Tch).

\begin{tabular}{|c|c|c|c|c|c|c|c|c|c|c|}
\hline May 2009 & & $\mathrm{Aj}$ & Ap & $\mathrm{Lr}$ & $\mathrm{Lp}$ & $\mathrm{G} 2$ & Gx & Le & $\mathrm{Sp}$ & Tch \\
\hline \multirow{3}{*}{ D. albomicans } & Banana & $4 *$ & 217 & 220 & 626 & 0 & 0 & 0 & 0 & 0 \\
\hline & Fig & 0 & 47 & 23 & 490 & 2 & 0 & 8 & 10 & 4 \\
\hline & Mixed vegetables & 0 & 29 & 31 & 0 & 0 & 0 & 0 & 0 & 5 \\
\hline \multirow{3}{*}{ D. takahashi } & Banana & 1 & 0 & 0 & 0 & 0 & 0 & 0 & 0 & 0 \\
\hline & Fig & 0 & 0 & 0 & 0 & 0 & 0 & 0 & 0 & 0 \\
\hline & Mixed vegetables & 4 & 0 & 0 & 0 & 0 & 0 & 0 & 0 & 0 \\
\hline \multirow{4}{*}{ D. lacteicornis } & Cherry & 0 & 0 & 0 & 0 & 0 & 5 & 0 & 0 & 0 \\
\hline & Banana & 0 & 0 & 0 & 0 & 0 & 0 & 0 & 0 & 0 \\
\hline & Fig & 0 & 0 & 0 & 0 & 0 & 0 & 0 & 0 & 0 \\
\hline & Mixed vegetables & 0 & 0 & 0 & 0 & 0 & 0 & 0 & 0 & 6 \\
\hline \multicolumn{11}{|l|}{ September 2009} \\
\hline \multirow{3}{*}{ D. albomicans } & Banana & 0 & 0 & 90 & 0 & 0 & 0 & 0 & 0 & 0 \\
\hline & Cherry & 0 & 1 & 11 & 5 & 0 & 0 & 0 & 0 & 0 \\
\hline & Mixed vegetables & 0 & 2 & 16 & 0 & 0 & 0 & 0 & 0 & 0 \\
\hline \multirow{3}{*}{ D. quadrilineata } & Banana & 0 & 0 & 0 & 0 & 0 & 0 & 0 & 0 & 0 \\
\hline & Cherry & 0 & 0 & 0 & 0 & 0 & 0 & 0 & 0 & 0 \\
\hline & Mixed vegetables & 0 & 0 & 0 & 0 & 0 & 0 & 0 & 0 & 0 \\
\hline \multirow{3}{*}{ D. daruma } & Banana & 0 & 0 & 0 & 0 & 0 & 0 & 0 & 0 & 0 \\
\hline & Cherry & 0 & 0 & 0 & 1 & 0 & 0 & 0 & 0 & 0 \\
\hline & Mixed vegetables & 7 & 0 & 11 & 59 & 0 & 0 & 0 & 0 & 0 \\
\hline
\end{tabular}

* possible misidentification of host pupae.

each sampling occasion traps were set approximately at the same locations (Fig. 1). In December 2007, additional traps were set at Funauki. Survey periods and numbers of traps used on each occasion are given in Table 1. After 5-7 days the traps were collected, and brought back to the laboratory. When the host individuals in the traps pupated they were collected identified to species according to Okada (1968) and placed in a separate Petri dish containing wet tissue paper. In several cases pupae of closely related species could not be identified based on their morphology (e.g. Drosophila bipectinata Duda - D. ananassae Doleschall and D. albomicans Duda - D. ruberrima de Meijere). Flies and wasps that emerged from the pupae were counted and the species identified. Two wasp species, Leptopilina victoriae Nordlander and L. ryukyuensis Novković \& Kimura, were difficult to identify based on their morphology and they were identified using molecular data (Novković et al., 2011) and a knowledge of their ecology, as these two species emerge from different host species. In the surveys of December 2007 and May 2009, flies emerged before the pupae were collected and therefore fly mortality, and in the case of May 2009 the total number of pupae could not be determined. To determine if there are different host-parasitoid communities in different habitats, supplementary collections were carried out in May and September 2009 using traps baited with cherry and mixed vegetables (mashed onion, potato and spinach). In May 2009 naturally-occurring figs were collected and brought back to the laboratory where the parasitism of the drosophilids breeding in these figs was also determined.

Based on the data from banana traps, the seasonality and habitat differences of the 3 most frequently recorded species of flies were examined using ANOVA and a post hoc Tukey test. Data from September 2009 was excluded from the statistical analyses because the numbers of insects emerging from the dif- ferent traps were pooled. All analyses were performed in Jmp v6.1 (SAS Institute, Cary, NC, USA).

\section{Host association}

Host association was experimentally analyzed using laboratory strains of four common wasps (A. japonica, A. pleuralis (Ashmead), L. ryukyuensis and L. pacifica Novković \& Kimura) and nine common drosophilids (D. takahashii Sturtevant, $D$. ficusphila Kikkawa \& Peng, D. lacteicornis Okada, D. ananassae, D. bipectinata, D. albomicans, D. ruberrima, D. quadrilineata de Meijere and $D$. daruma Okada). These strains originated from individuals collected by H. Mitsui on Iriomote-jima using banana-baited traps between $22^{\text {nd }}$ and $29^{\text {th }}$ March 2006. Other parasitoids and/or flies were rarely sampled and/or difficult to rear. All drosophilid strains were reared in vials containing cornmeal-malt medium. The strains of L. ryukyuensis, A. pleuralis and $A$. japonica were maintained using $D$. simulans as the host. The strain of L. pacifica, which could not be reared on $D$. simulans, was reared on D. sulfurigaster albostrigata Wheeler. D. simulans and D. sulfrigaster were chosen as the hosts in order to avoid the effect of adaptation, since they do not naturally occur on Iriomote-jima. The wasp strains were maintained for two or three generations in the laboratory $(15 \mathrm{~L}: 9 \mathrm{D}$ at $23^{\circ} \mathrm{C}$ ) prior to the experiments. All the wasps used in the experiments were 4-11 days old, mated, allowed to feed on drosophila medium and experienced on patches of $D$. simulans or $D$. sulfrigaster larvae.

\section{Host acceptance}

To determine if parasitoids accept the larvae of different drosophilids as hosts, 30 to 40 second instar drosophilid larvae were placed in a Petri dish ( $3 \mathrm{~cm}$ in diameter) containing a small amount of Drosophila medium. Five female wasps were introduced into each Petri dish and left to oviposit for $4 \mathrm{~h}$. After the 

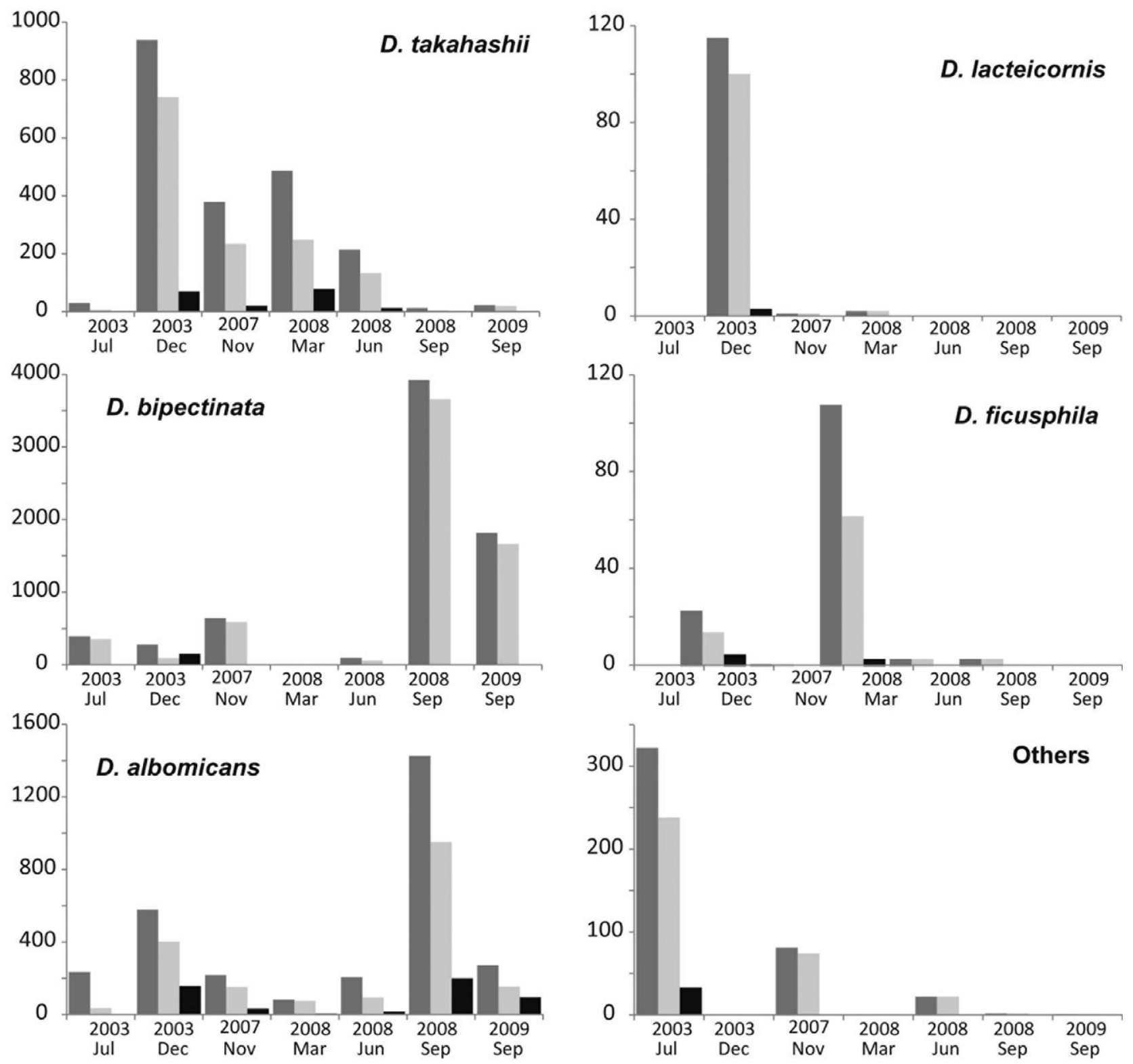

Fig. 2. Number of pupae collected (dark grey) and number of flies (light grey) and wasps (black) that emerged from the pupae.

removal of the wasps, fly larvae were dissected and checked for the presence/absence of wasp eggs. The percentage oviposition was calculated as the number of parasitized larvae per total number of larvae.

\section{Host suitability}

Host suitability for the different wasp species was determined as follows. Two-day-old fly larvae were placed in a Petri dish containing a small amount of rearing medium and then exposed to several (2-5) female wasps, whose oviposition behaviour was followed under a stereoscopic microscope. Characteristic oviposition behaviour, such as full extension of the ovipositor after contact with a host (van Lenteren, 1976; van Lenteren et al., 1998 ) and longer insertions of the ovipositor into larvae (>10 s) (Vet \& Bakker, 1985; Visser, 1995; Dubuffet et al., 2006) were taken as indicators of successful oviposition. When oviposition was confirmed, parasitized fly larvae were transferred into vials containing Drosophila medium. The vials were later checked for the emergence of flies and/or wasps. Approximately 30 larvae of each fly species were parasitized. In some cases, few or no parasitized larvae were obtained, due to low host acceptance.

In the field, $L$. victoriae was observed to parasitize $D$. bipectinata but we did not have a laboratory strain of this parasitoid species collected from Iriomote-jima. The incidence of parasitism by this parasitoid was determined using L. victoriae strains that originated from Kota-Kinabalu, Malaysia (collected in March 2008 by M. Kondo; $6^{\circ} \mathrm{N}, 116^{\circ} \mathrm{E}$ ) and Bogor, Indonesia (collected in June 2008 by A. Suwito; $6.6^{\circ} \mathrm{S}, 106.8^{\circ}$ E) (Fig. 1). To check for the existence of local differences in host resistance/parasitoid virulence, laboratory strains of $D$. bipectinata from Kota-Kinabalu (collected in March 2008 by M. Kondo) and Bogor (collected in June 2008 by A. Suwito) were also used. All experiments were carried out at $23^{\circ} \mathrm{C}$.

\section{RESULTS}

\section{Species composition and abundance}

Fifteen drosophilid and 12 parasitoid species were recorded in the field survey (Tables 2 and 3). Both host 

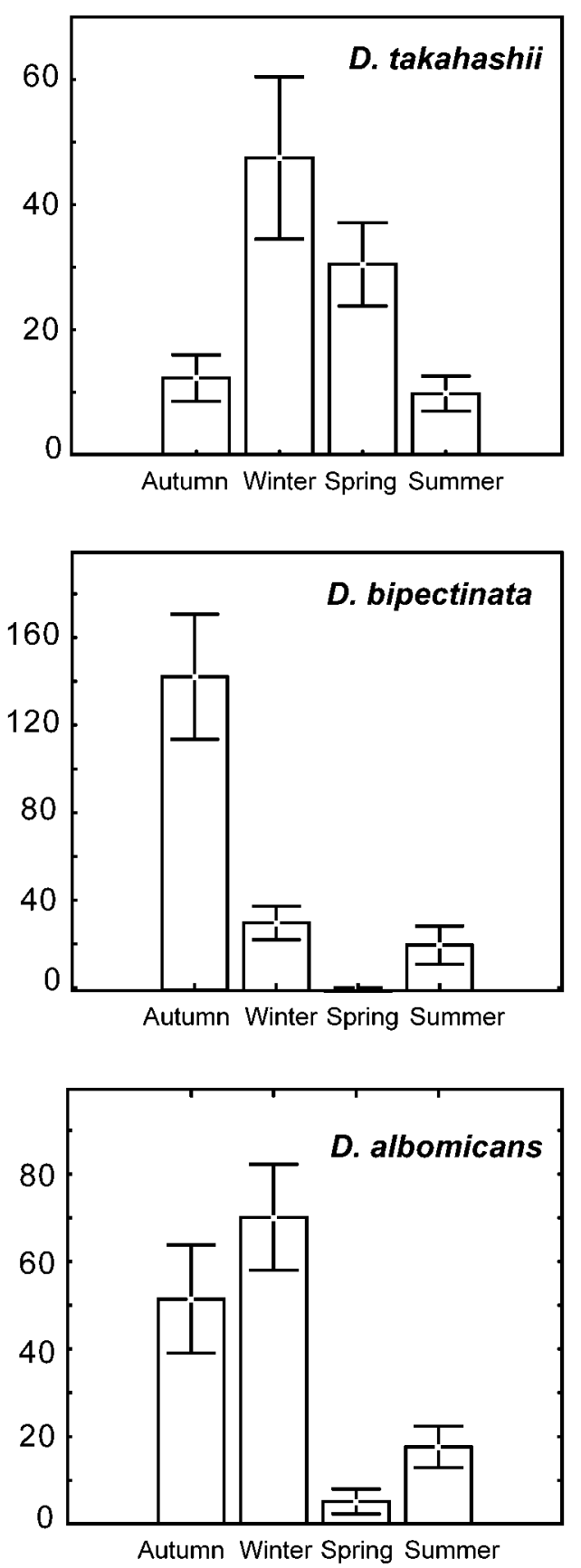

Fig. 3. Average number of pupae of D. takahashii, D. bipectinata and D. albomicans collected from each trap $(30 \mathrm{~g}$ of banana bait) in the different seasons. Error bars represent standard error.

and parasitoid species differed greatly in their overall and seasonal abundance. The most abundant drosophilid species breeding in banana baits were D. bipectinata, $D$. takahashii and D. albomicans (Fig. 2). D. takahashii was significantly more abundant in winter than summer and autumn $\left(\mathrm{F}_{(3,102)}=4.17, P=0.008\right)$, D. albomicans was significantly more abundant in winter than summer or spring $\left(\mathrm{F}_{(3,102)}=6.24, P<0.001\right)$, while $D$. bipectinata was significantly more abundant in autumn $\left(\mathrm{F}_{(3,102)}=\right.$ 11.47, $P<0.001$ ) (Fig. 3).

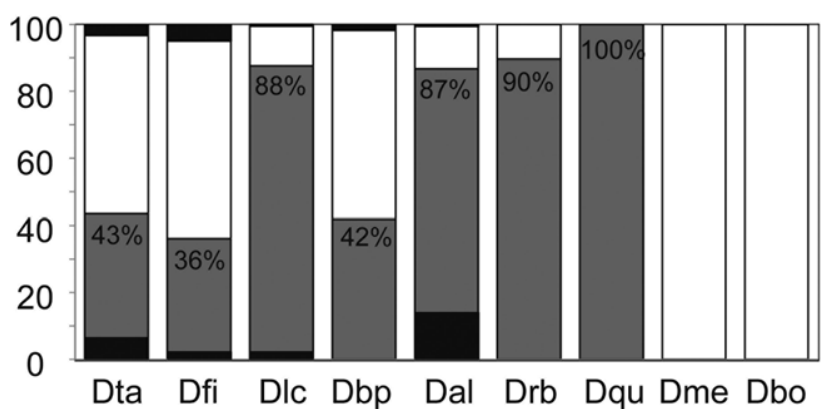

Fig. 4. Number of each of the drosophilids recorded in the traps set in the forest (grey) and domestic (white) environments, and the percentage parasitized (black). Percentage parasitism expressed per gram of banana bait. D. takahashii (Dta), D. ficusphila (Dfi), D. lacteicornis (Dlc), D. bipectinata (Dbp), D. albomicans (Dal), D. ruberrima (Drb), D. quadrilineata (Dqu), D. melanogaster (Dme) and D. bocki (Dbo).

$D$. bipectinata and D. takahashii were present in both forest and domestic areas, while $D$. albomicans was more abundant in forest $\left(\mathrm{F}_{(1,104)}=21.92, P<0.001\right)$. Species abundant in forest included $D$. ruberrima, D. lacteicornis and $D$. quadrilineata, while D. ficusphila, D. melanogaster and $D$. bocki Baimai were predominantly found in domestic environments (Fig. 4). Other drosophilid species recorded were $D$. ananassae, $D$. daruma, D. sternopleuralis Okada, Scaptodrosophila bryani (Malloch), S. dorsocentralis (Okada) and Phortica magna (Okada). Nine of these 15 species were attacked by parasitoids in the field (Table 2).

The parasitoid species that were most frequently recorded emerging from drosophilid pupae collected using banana-baited traps were Asobara japonica, A. pleuralis (Braconidae), Leptopilina ryukyuensis and $L$. pacifica (Figitidae). L. victoriae was recorded only in December 2003. Ganaspis xanthopoda, Ganaspis sp. IR1, Ganaspis sp. IR2 (Figitidae) and Trichopria sp. (Diapriidae) were also recorded.

Asobara japonica mainly emerged from D. takahashii pupae and A. pleuralis, L. ryukyuensis and L. pacifica mainly from $D$. albomicans pupae. $L$. victoriae emerged only from $D$. bipectinata pupae. The overall percentage parasitism ranged from $3.7 \%$ in July 2003 to $20.1 \%$ in December 2003. The percentage parasitism of each species of host ranged from 0 to $54.3 \%$. The maximum percentage parasitism was of $D$. bipectinata pupae in December 2003 (Table 2). D. albomicans was the only species that was parasitized throughout the season, with the highest percentage parasitism of $34.8 \%$ recorded in September 2009. For D. takahashii the highest percentage parasitism recorded was $16.2 \%$ in March 2008 . The percentage parasitism was higher in forest than in domestic environments.

In the supplementary collections, which were carried out in May 2009 (Table 3), L. pacifica was the most abundant parasitoid emerging from $D$. albomicans pupae collected from both banana-baited traps and naturally occurring figs. L. ryukyuensis and A. pleuralis were also 


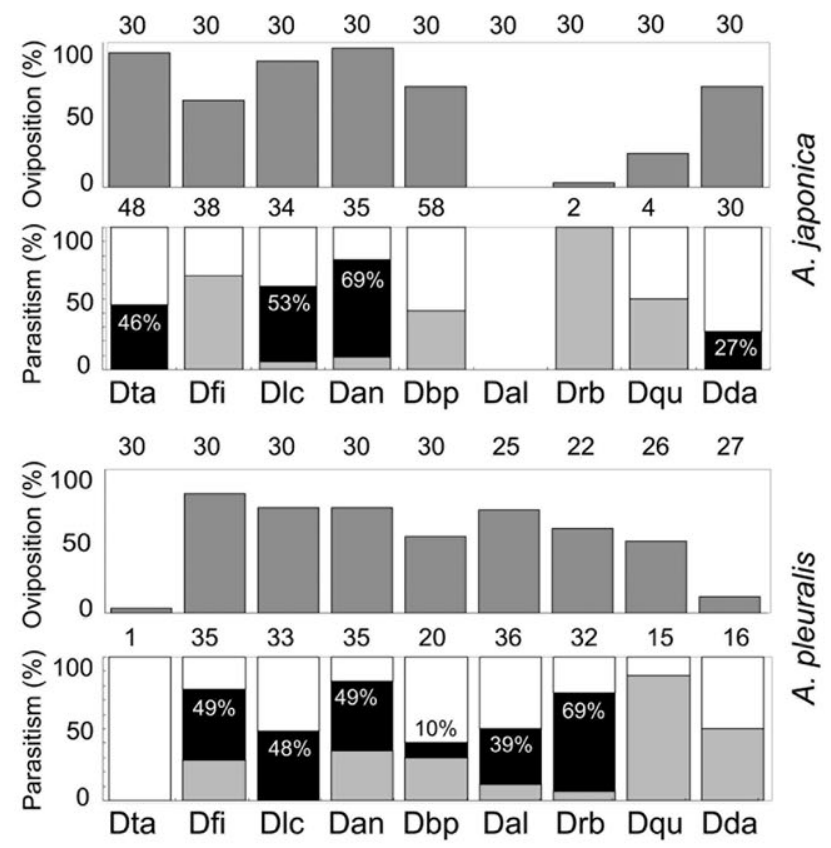

TABLE 4. Percentage parasitism of D. bipectinata by L. victoriae in the laboratory experiments.

\begin{tabular}{|c|c|c|c|c|c|c|c|c|}
\hline \multirow[b]{3}{*}{ D. bipectinata } & \multicolumn{8}{|c|}{ Leptopilina victoriae } \\
\hline & \multicolumn{4}{|c|}{ BG } & \multicolumn{4}{|c|}{ KK } \\
\hline & $\mathrm{N}$ & $\mathrm{F}$ & $\mathrm{W}$ & $\mathrm{D}$ & $\mathrm{N}$ & $\mathrm{F}$ & $\mathrm{W}$ & $\mathrm{D}$ \\
\hline Bogor (BG) & 33 & 15 & 0 & 18 & 30 & 20 & 1 & 9 \\
\hline Kota Kinabalu (KK) & 30 & 23 & 1 & 6 & 30 & 22 & 1 & 7 \\
\hline Iriomote-jima (IR) & 30 & 27 & 0 & 3 & 60 & 4 & 36 & 20 \\
\hline
\end{tabular}

$\mathrm{N}$ - parasitized larvae; $\mathrm{F}$ - emerged flies; $\mathrm{W}$ - emerged wasps; $\mathrm{D}-$ dead individuals.

was low in D. albomicans, D. ruberrima and D. quadrilineata - the three species belonging to the immigrans species group. On the other hand, the percentage oviposition of A. japonica in D. ficusphila and D. bipectinata was relatively high, but the percentage survival of their offspring in these two species was $0 \%$. Both A. pleuralis and $L$. ryukyuensis either did not survive or the percentage survival was low in D. bipectinata and D. quadrilineata. $L$. ryukyuensis had the widest potential-host range, successfully parasitizing 6 of the 9 species offered. $L$. pacifica readily oviposited in $D$. daruma and all three species of the immigrans species group and successfully developed in all four of these species. The percentage oviposition of this parasitoid in other drosophilid species was low.

Table 4 shows the results of the experiments performed on the strains of L. victoriae from Kota-Kinabalu and Bogor using D. bipectinata from these two localities and Iriomote-jima. $D$. bipectinata strains from Kota- Kinabalu and Bogor were resistant to both of the strains of $L$. victoriae tested. However, although resistant to the $L$. victoriae strain from Bogor, the D. bipectinata strain from Iriomote-jima was highly susceptible to the $L$. victoriae strain from Kota-Kinabalu, confirming the existence of geographic variation in host resistance and/or parasitoid virulence.

\section{DISCUSSION}

\section{Host-parasitoid community recorded on Iriomote-jima}

Fig. 5. Percentage oviposition and percentage parasitism for the abundant drosophilid species: D. takahashii (Dta), D. ficusphila (Dfi), D. lacteicornis (Dlc), D. ananassae (Dan), D. bipectinata (Dbp), D. albomicans (Dal), D. ruberrima (Drb), D. quadrilineata (Dqu) and D. daruma (Dda). Number of individuals used in the experiments is given above the columns. In the graphs of the percentage parasitism, emerged flies are shown in grey, dead flies in white and emerged wasps in black. Percentage successfully parasitized is indicated by the numbers inside the columns that are associated with the black bars.

abundant. L. ryukyuensis was the most abundant parasitoid in September 2009.

\section{Host associations}

Fig. 5 shows the percentage oviposition and percentage parasitism recorded in the laboratory experiments. $A$. japonica did not oviposit or the percentage oviposition

A complex host-parasitoid community consisting of at least 9 frugivorous drosophilid hosts and 12 parasitoid wasp species was recorded on this subtropical island. Drosophila bipectinata, D. albomicans and D. takahashii were the major drosophilids breeding in the banana baits on Iriomote-jima, accounting for more than $97 \%$ of all the drosophilids. In the survey conducted on this island by Hirai et al. (2000) in 1994-1995, these three species made up no more than $43 \%$ of all the drosophilids that bred in naturally occurring fruit and only $23 \%$ of the adult flies collected from banana-baited traps. The dominance of these three species in our survey was due to the fact that our collection consisted only of the species that bred in banana-baits. Other drosophilid species recorded by Hirai et al. (2000) may rarely breed in banana-like succulent fruit. Thus although the results given here are biased, they are standardized and facilitate comparison between 
seasons, environments and similar bait-based surveys at other locations.

The three drosophilid species most abundant on Iriomote-jima, D. bipectinata, D. albomicans and $D$. takahashii, seldom occur in central Japan. Likewise, frequent species obtained using banana-baited traps in central Japan, e.g. D. lutescens, D. immigrans, D. simulans and $D$. auraria, were not recorded in this study (Beppu, 2006; Mitsui \& Kimura, 2010). The distribution of these drosophilids is most likely related to their susceptibility to cold (Kimura, 2004). Compared to species recorded in central Japan, species occurring on Iriomote-jima have low cold tolerance (Kimura, 2004), which limits their northernmost distribution. On the other hand, due to trade-offs related to cold tolerance, temperate species are expected to be competitively inferior to subtropical species in warmer areas, which limits their southernmost distributions. Cold tolerant species are reported to have lower walking speeds and slower pupal development compared to their cold-intolerant counter-parts, which might render them more susceptible to predation and parasitism in warmer areas (Shiota \& Kimura, 2007). Additionally, a trade-off was found between heat and cold tolerance in D. melanogaster, but has yet to be confirmed for other drosophilid species (Anderson et al., 2003).

On Iriomote-jima, D. bipectinata was abundant from summer to early winter, D. albomicans from early summer to early spring (also see Hirai et al., 2000) and $D$. takahashii from late autumn to early summer. The seasonality of these species can also partly be explained by their thermal tolerance. D. takahashii is the most cold tolerant and increases in numbers from mid-winter, while $D$. bipectinata is the most heat tolerant and cold intolerant (Kimura, 2004; Novković, unpubl. data) and increases in numbers from early summer. Abundance of these drosophilids is probably further influenced by the amount of rainfall and seasonal differences in the availability of fruit, but their effects are unknown.

Twelve parasitoid species belonging to 7 genera and 5 families were recorded from Iriomote-jima, six of them new to this island. Species of the genera Asobara, Leptopilina, Ganaspis and Leptolamina are larval parasitoids, while those of Trichopria and Spalangia are pupal parasitoids (Carton et al., 1986; Mitsui et al., 2007). To our knowledge, this is the first report of a Tachinaephagus species parasitizing drosophilids. The developmental stage of the host that Tachinaephagus sp. oviposits in is not known. The most frequently collected parasitoid species in the field were A. japonica, A. pleuralis, $L$. ryukyuensis and L. pacifica. Pupal parasitoids were rarely collected in this survey because most of the drosophilid individuals in the baits were larvae at the time of collection.

In December 2003, a high parasitism of D. bipectinata by $L$. victoriae was recorded. However, this wasp was not recorded before or after this occasion, despite the abundance of $D$. bipectinata. Consequently, this wasp species might have been introduced to this island shortly before the samples were collected in December 2003, became abundant by exploiting the low-resistant $D$. bipectinata host and then possibly became extinct. This hypothesis is supported by the fact that the population of $D$. bipectinata on Iriomote-jima declines in size in winter and spring (also see Hirai et al., 2000) due to its low cold tolerance. Therefore, it would be difficult for $L$. victoriae to survive the cold season.

With at least 9 frugivorous drosophilid hosts and 12 parasitoid wasps, the Iriomote-jima host-parasitoid community is more diversified and complex than other Drosophila-parasitoid communities exploiting decaying fruit recorded in temperate areas such as central Japan, southern France and Netherlands (Janssen et al., 1988; Allemand et al., 1999; Mitsui \& Kimura, 2010). While $A$. japonica and $G$. xanthopoda are the dominant species on the main islands of Japan (Mitsui et al., 2007; Mitsui \& Kimura, 2010) there is a more diversified parasitoid community on Iriomote-jima, including abundant species such as $A$. pleuralis, L. ryukyuensis and L. pacifica.

Percentage parasitism of some drosophilid hosts reaches $46 \%$ in the Tokyo area (Mitsui \& Kimura, 2010). Average percentage parasitism recorded in summer in Europe ranges from $5 \%$ to $40 \%$ in mid-summer, reaching $80 \%$ in some habitats (Fleury et al., 2009). On Iriomotejima the average percentage parasitism was $9.1 \%$. However, large seasonal variations were recorded. A relatively high percentage parasitism of D. bipectinata by L. victoriae was recorded in December 2003 (54\%), of $D$. albomicans in September 2009 (35\%) and of D. takahashii by A. japonica in March 2008 (16.2\%). Such seasonal variations in percentage parasitism are probably related to the seasonal variations in host abundance with the parasitoids increasing in numbers following the increase in the number of their hosts. Seasonality in host abundance in turn, as previously discussed, seems to be related to their cold tolerance. While on Iriomote-jima drosophilids are active throughout the season, in the temperate regions, the activity of most drosophilid flies is more or less limited to the warmer seasons and temperate parasitoids are also mainly active in summer, i.e., the season when drosophilid flies are most active. Parasitoids on Iriomote-jima, however, seem to be active throughout the year.

\section{Host associations}

On Iriomote-jima, A. japonica mainly emerged from $D$. takahashii and occasionally from $D$. ananassae and $D$. daruma. In central Japan, this parasitoid parasitizes most of the species of Drosophila it encounters (Ideo et al., 2008; Mitsui et al., 2010). On Iriomote-jima, however, our results indicate that there are several species that are completely resistant to this wasp (e.g. D. ficusphila and $D$. bipectinata). The presence of drosophilid species resistant to $A$. japonica may result from longer evolutionary interactions between $A$. japonica and potential hosts on subtropical Iriomote-jima than mainland Japan. Based on the fact that the main-island populations are parthenogenetic whereas the Iriomote-jima population reproduces sexually, it is assumed that this species origi- 
nated in the subtropics and that its recent colonization of temperate regions is associated with the evolution of parthenogenesis (Murata et al., 2009).

Based on the results of the laboratory experiments, resistance against one parasitoid did not provide resistance against other parasitoid species. For example, $D$. takahashii was resistant to L. pacifica but susceptible to A. japonica, D. albomicans was resistant to $A$. japonica but was susceptible to the other three wasp species used in these experiments. In other words, the resistance/virulence mechanisms in these drosophilids and their parasitoids are species-specific, implying that these drosophilids and parasitoids have been subjected to complex coevolutionary interactions. Furthermore, most parasitoid species included at least one of the three major Drosophila species in their host range, implying that the abundance of potential hosts is one of the factors important in the evolution of parasitoid host use.

A disparity between host acceptance and host suitability was observed. Wasps oviposited in drosophilids in which they were unable to develop (e.g. A. japonica in D. ficusphila and D. bipectinata, L. ryukyuensis in D. bipectinata). While the evolution of a preference to oviposit in suitable hosts increases their parasitic efficiency, a preference for ovipositing in a wide range of hosts could facilitate switching from one host to another - when the preferred host becomes scarce or develops a high resistance towards the parasitoid.

The experiments with $L$. victoriae and $D$. bipectinata from Kota Kinabalu, Bogor and Iriomote-jima revealed a geographic variation in host resistance and/or parasitoid virulence. D. bipectinata from Kota Kinabalu and Bogor were resistant to the two strains of $L$. victoriae. D. bipectinata from Iriomote-jima, however, was resistant to $L$. victoriae from Bogor, but not from Kota Kinabalu. Thus, both $L$. victoriae and $D$. bipectinata showed intra-specific geographic variation, probably as a result of local-specific interactions.

In this survey, we explored the diversity, abundance and host association of frugivorous drosophilids and their parasitoids breeding in banana baited traps on Iriomotejima. We also recorded the seasonal and habitat differences of the different species. Most parasitoids exhibited species-specific interactions with more than one host species, suggesting complex coevolutionary interactions. To broaden our understanding of the host-parasitoid community on this island, it would be beneficial to study other breeding substrates such as fungi and decaying plant material. Furthermore, the influence of rainfall and availability of natural fruit on host-parasitoid community dynamics is a subject yet to be explored. Finally, it would be interesting to broaden the survey to other subtropical/tropical areas, in order to gain a more comprehensive knowledge of the structure and complexity of interactions in tropical host-parasitoid communities.

ACKNOWLEDGEMENTS. We wish to sincerely thank $\mathrm{K}$. Kamijo and Y. Higashiura for identifying some of the wasps and A. Suwito and M. Kondo for collecting samples. This work was supported by a Grant-in-Aid from the Ministry of Education, Science, Sports and Culture of Japan (No. 23370005).

\section{REFERENCES}

Allemand R., Fleury F., Lemaître C. \& Boulétreau M. 1999: Dynamique des populations et interactions compétitives chez deux espèces de Leptopilina, parasitoïdes de drosophiles, dans la vallée du Rhône (Hymenoptera: Figitidae). Ann. Soc. Entomol. Fr. 35: 97-103.

Anderson A.R., Collinge J.E., Hoffmann A.A., Kellett M. \& McKechnie S.W. 2003: Thermal tolerance trade-offs associated with the right arm of chromosome 3 and marked by the hsr-omega gene in Drosophila melanogaster. Heredity 90: 195-202.

BEPPU K. 2006: Seasonal change of drosophilid assemblage and adult age structure of the common drosophilid species in the Imperial Palace grounds, Tokyo. Mem. Nat. Sci. Mus. 43: 295-334 [in Japanese, English abstr.].

Boulétreau M. \& FoullLet P. 1982: Variabilité génétique intrapopulation de l'adéquation de Drosophila melanogaster à un de ses parasites hyménoptères. C. R. Acad. Sci. Paris 295: 775-778.

CARTON Y. \& NAPPI A. 1991: The Drosophila immune reaction and the parasitoid capacity to evade it: Genetic and coevolutionary aspects. Acta Oecol. 12: 89-104.

Carton Y., Boulétreau M., van Alphen J.J.M. \& van Lenteren J.C. 1986: The Drosophila parasitic wasps. In Ashburner M., Carson H.L. \& Thompson J.N. (eds): The Genetics and Biology of Drosophila. Vol. 3e. Academic Press, London, pp. 347-394.

Carton Y., Haouas S., Marrakchi M. \& Hochberg M. 1991: Two competing parasitoid species coexist in sympatry. Oikos 60: $222-230$.

Dubuffet A., Rodriguez Alvarez C.I., Drezen J.M., van Alphen J.J.M. \& Poirié M. 2006: Do parasitoid preferences for different host species match virulence? Physiol. Entomol. 31: 170-177.

Fleury F., Ris N., Allemand R., Fouillet P., Carton Y. \& BoulÉtreAu M. 2004: Ecological and genetic interactions in Drosophila-parasitoids communities: a case study with D. melanogaster, D. simulans and their common Leptopilina parasitoids in south-eastern France. Genetica 120: 181-194.

Fleury F., Gilbert P., Ris N. \& Allemand R. 2009: Ecology and life history evolution of frugivorous Drosophila parasitoids. Adv. Parasitol. 70: 3-44.

Jansen A., Driessen G., De HaAn M. \& Roodbol N. 1988: The impact of parasitoids on natural populations of temperate woodland Drosophila. Neth. J. Zool. 38: 61-73.

HARDY I.C.W. \& GodfraY H.C.J. 1990: Estimating the frequency of constrained sex allocation in field populations of Hymenoptera. Behaviour 114: 1-4.

HertLein M.B. 1986: Seasonal development of Leptopilina boulardi (Hymenoptera, Eucoilidae) and its hosts, Drosophila melanogaster and Drosophila simulans (Diptera, Drosophilidae) in California. Environ. Entomol. 15: 859-866.

Hirai Y., Goto S.G., Yoshida T. \& Kimura M.T. 2000: Faunal and ecological surveys on drosophilid flies in Iriomote-jima, a subtropical island of Japan. Entomol. Sci. 3: 273-284.

Ideo S., Watada M., Mitsui H. \& Kimura M.T. 2008: Host range of Asobara japonica (Hymenoptera: Braconidae), a larval parasitoid of drosophilid flies. Entomol. Sci. 11: 1-6.

Kimura M.T. 2004: Cold and heat tolerance of drosophilid flies with reference to their latitudinal distributions. Oecologia 140: 442-449. 
KraAiJeveld A.R. \& van AlPhen J.J.M 1994: Geographical variation in resistance of the parasitoid Asobara tabida against encapsulation by Drosophila melanogaster larvae: the mechanism explored. Physiol. Entomol. 19: 9-14.

KRAAIJEVELD A.R. \& VAN AlPhen J.J.M. 1995: Geographical variation in encapsulation ability of Drosophila melanogaster larvae and evidence for parasitoid-specific components. Evol. Ecol. 9: 10-17.

KraaiJeveld A.R. \& Godfray H.C.J. 1999: Geographical patterns in the evolution of resistance and virulence in Drosophila and its parasitoids. Am. Nat. 153: S61-74.

KraAiJeveld A.R., van Alphen J.J.M. \& Godfray H.C.J. 1998: The coevolution of host resistance and parasitoid virulence. Parasitology 166: S29-45.

Mitsui H. \& KimuRa M.T. 2010: Distribution, abundance and host-association of two parasitoid species attacking frugivorous drosophilid larvae in central Japan. Eur. J. Entomol. 107: 535-540.

Mitsui H., van Achterberg K., Nordlander G. \& Kimura M.T. 2007: Geographical distributions and host associations of larval parasitoids of frugivorous Drosophilidae in Japan. $J$. Nat. Hist. 41: 1731-1738.

Murata Y., Ideo S., Watada M., Mitsui H. \& Kimura M.T. 2009: Genetic and physiological variation among sexual and parthenogenetic populations of Asobara japonica (Hymenoptera: Braconidae), a larval parasitoid of drosophilid flies. Eur. J. Entomol. 106: 171-178.

Novković B., Mitsui H., Suwito A. \& Kimura M.T. 2011: Taxonomy and phylogeny of Leptopilina species (Hymenoptera: Cynipoidea, Figitidae) attacking frugivorous drosophilid flies in Japan, with description of three new species. Entomol. Sci. 14: $333-346$.

OKadA T. 1965: Drosophilidae of the Okinawa islands. Kontyu 33: $327-350$.
OкADA T. 1968: Systematic Study of the Early Stages of Drosophilidae. Bunka Zugeisha, Tokyo, 188 pp.

Shiota H. \& Kimura M.T. 2007: Evolutionary trade-offs between thermal tolerance and locomotor and developmental performance in drosophilid flies. Biol. J. Linn. Soc. 90: 375-380.

TAKada H. \& Makino H. 1981: A Drosophila survey of Iriomote island, Okinawa, Japan. J. Fac. Gen. Educ. Sapporo Univ. 19: $27-36$.

VAN LENTEREN J.C. 1976: The development of host discrimination and the prevention of superparasitism in the parasite Pseudeucoila bochei (Hym.: Cynipidae). Neth. J. Zool. 26: $1-83$.

VAN Lenteren J.C., Isidoro N. \& Bin F. 1998: Functional anatomy of the ovipositor clip in the parasitoid Leptopilina heterotoma (Thompson) (Hymenoptera: Eucoilidae), a structure to grip escaping host larvae. Int. J. Insect Morphol. Embryol. 27: 263-268.

Vet L.E.M. \& BAKKeR K. 1985: A comparative functional approach to the host detection behavior of parasitic wasps. II. A quantitative study on eight eucoilid species. Oikos 44: 487-489.

VISSER M.E. 1995: The effect of competition on oviposition decisions of Leptopilina heterotoma (Hymenoptera: Eucoilidae). Anim. Behav. 49: 1677-1687.

YoRozuYa H. 2006: Effects of parasitoids on a mycophagous drosophilid community in northern Japan and an evaluation of the disproportionate parasitism hypothesis. Entomol. Sci. 9: $13-22$.

Wertheim B., Allemand R., Vet L.E.M. \& Dicke M. 2006: Effect of aggregation pheromone on individual behaviour and food web interactions: a field study on Drosophila. Ecol. Entomol. 31: 216-226.

Received December 20, 2011; revised and accepted May 29, 2012 